Claremont Colleges

Scholarship@ Claremont

All HMC Faculty Publications and Research

HMC Faculty Scholarship

6-24-2009

\title{
Sum Rules and Universality in Electron-modulated Acoustic Phonon Interaction in a Free-standing Semiconductor Plate
}

Shigeyasu Uno

Nagoya University

Darryl H. Yong

Harvey Mudd College

Nobuya Mori

Osaka University

\section{Recommended Citation}

Uno, S, Yong, DH, Mori, N. Sum Rules and Universality in Electron-modulated Acoustic Phonon Interaction in a Free-standing Semiconductor Plate. Phys Rev B. 2009;79(23): 1-7.

This Article is brought to you for free and open access by the HMC Faculty Scholarship at Scholarship @ Claremont. It has been accepted for inclusion in All HMC Faculty Publications and Research by an authorized administrator of Scholarship @ Claremont. For more information, please contact scholarship@cuc.claremont.edu. 


\title{
Sum rules and universality in electron-modulated acoustic phonon interaction in a free-standing semiconductor plate
}

\author{
Shigeyasu Uno* \\ Department of Electrical Engineering and Computer Science, Graduate School of Engineering, Nagoya University, Furo-cho, \\ Chikusa-ku, Nagoya 464-8603, Japan \\ Darryl Yong \\ Department of Mathematics, Harvey Mudd College, 301 Platt Boulevard, Claremont, California 91711, USA \\ Nobuya Mori \\ Department of Electronic Engineering, Osaka University, 2-1 Yamada-oka, Suita, Osaka 565-0871, Japan
}

(Received 22 April 2009; published 24 June 2009)

\begin{abstract}
Analysis of acoustic phonons modulated due to the surfaces of a free-standing semiconductor plate and their deformation-potential interaction with electrons are presented. The form factor for electron-modulated acoustic phonon interaction is formulated and analyzed in detail. The form factor at zero in-plane phonon wave vector satisfies sum rules regardless of electron wave function. The form factor is larger than that calculated using bulk phonons, leading to a higher scattering rate and lower electron mobility. When properly normalized, the form factors lie on a universal curve regardless of plate thickness and material.
\end{abstract}

DOI: 10.1103/PhysRevB.79.235328

PACS number(s): 72.10.Di

\section{INTRODUCTION}

Acoustic phonons in nanoscale devices differ from bulk phonons due to mechanical mismatch, as reported for freestanding plates, ${ }^{1-3} \quad$ GaAs/AlAs and GaN/AlN heterostructures, ${ }^{4-8} \mathrm{Si} / \mathrm{SiO}_{2}$ structures in advanced MOSFETs, ${ }^{9-11}$ and other structures. ${ }^{12}$ Incorporating those modulated phonons in electron-transport calculation is becoming important for device characteristic predictions. Among such nanoscale devices, free-standing (FS) plate is the simplest and most fundamental structure. A good understanding of the physics of electron-modulated acoustic phonon interactions in a free-standing plate will help the analysis of other heterostructures. Donetti et al. reported that modulated-acoustic-phonon-limited electron mobility in a FS Si plate is smaller than that calculated using bulk phonons. ${ }^{9}$ However, despite the simplicity of the structure, mechanisms of such reduction are not yet clear. Deeper analysis is required for better understanding of the phenomena and underlying physics.

In this work, insights into modulated acoustic phonons and their deformation-potential interaction with electrons in an ultrathin FS plate are reported. Electron-acoustic phonon interaction is formulated within the framework of Fermi's golden rule and elastic approximation. Modulated-acousticphonon wave functions are formally encapsulated into the form factor, so that the scattering rate and electron mobility are described in the same manner as the bulk phonon case. The form factor is studied both by analytical and numerical approaches. The analytical study of the form factor exhibits that sum rules hold at least for zero in-plane phonon wave number, and the numerical study reveals a universality in the form factor. The formulations and analytical study of the form factor are described in Sec. II, and the numerical study and discussions are presented in Sec. III. Finally, the conclusion follows in Sec. IV.

\section{THEORY}

\section{A. Modulated acoustic phonons and electron phonon interaction}

Assuming an isotropic and continuous material, modulated acoustic phonons in a free-standing plate are described by the Navier's equation in three dimensions,

$$
\rho \frac{\partial^{2} \boldsymbol{S}}{\partial t^{2}}=(\lambda+2 \mu) \nabla(\nabla \cdot S)-\mu \nabla \times(\nabla \times S),
$$

where $S$ is the displacement vector, $\rho$ is the mass density, $\lambda$ and $\mu$ are Lamé constants. Assuming a three-dimensional Cartesian coordinate system with $z$ axis normal to the plate, $S$ can be written as superposition of phonon normal modes

$$
\boldsymbol{S}(\boldsymbol{r}, t)=\sum_{q} C_{q} e^{-i \omega_{q} t} e^{i \boldsymbol{Q} \cdot \boldsymbol{R}} \boldsymbol{v}(z),
$$

where $\omega_{q}$ is the phonon energy, $C_{q}$ is a constant, and $\boldsymbol{R}$ $=(x, y)$ and $\boldsymbol{Q}=\left(q_{x}, q_{y}\right)$ are position and phonon wave vectors in $x-y$ plane, respectively. A three-dimensional vector $\boldsymbol{v}(z)$ represents the $z$ dependence of the normal modes. The traction-free boundary condition and symmetry consideration give an equation for determining allowed phonon modes. Resulting normal modes fall in two categories: shearhorizontal (SH) modes, which have only $y$ component assuming that the phonon wave travels in the $x$ direction, or mixed pressure-shear vertical (mixed P-SV) modes, which have $x$ and $z$ components and zero $y$ component. Only the mixed P-SV modes contribute to the acoustic deformationpotential (ADP) scattering, and they can be written as 


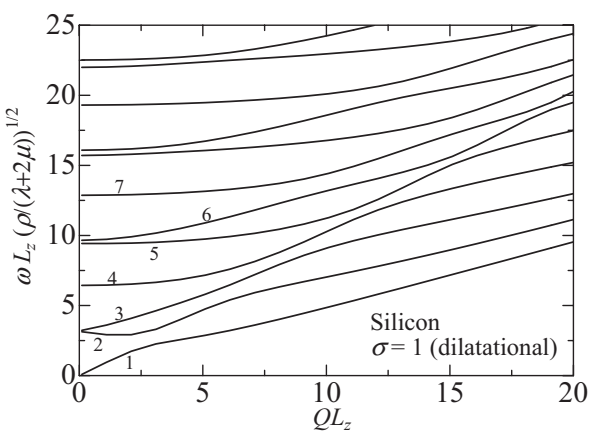

FIG. 1. Modulated phonon frequency $\omega$ as a function of in-plane phonon wave vector calculated for a free-standing Si plate. The vertical and horizontal axes are normalized so that the plot gives dispersion relations for arbitrary plate thickness, $L_{z}$. Numbers 1 through 7 are added to the lowest seven branches for explanation. The meaning of $\sigma$ is given in the text.

$$
\begin{aligned}
\boldsymbol{v}_{\mathrm{PSV}}(z)= & \frac{C_{l}}{\sqrt{q_{x}^{2}+q_{l}^{2}}}\left\{e^{i q_{l^{2}}}\left(\begin{array}{c}
q_{x} \\
0 \\
q_{l}
\end{array}\right)+\sigma e^{-i q_{l^{z}}}\left(\begin{array}{c}
q_{x} \\
0 \\
-q_{l}
\end{array}\right)\right\}, \\
& +\frac{C_{t}}{\sqrt{q_{x}^{2}+q_{t}^{2}}}\left\{e^{i q_{z^{2}}}\left(\begin{array}{c}
-q_{t} \\
0 \\
q_{x}
\end{array}\right)-\sigma e^{-i q_{l^{2}}}\left(\begin{array}{c}
q_{t} \\
0 \\
q_{x}
\end{array}\right)\right\},
\end{aligned}
$$

where $q_{l}$ and $q_{t}$ denote the $z$ components of longitudinal (pressure) and transverse (shear) phonon wave vectors, respectively. The coefficients $C_{l}$ and $C_{t}$ are related to phonon amplitude, and $\sigma$ takes either +1 (dilatational) or -1 (flexural). The origin of the $z$ axis is set at the middle of the plate thickness. The squared coefficients $\left|C_{l}\right|^{2}$ and $\left|C_{t}\right|^{2}$ can be written explicitly. ${ }^{3}$ However, allowed values of $q_{l}$ and $q_{t}$ for a given $q_{x}$ are determined by an implicit transcendental equation, ${ }^{1}$ so numerical calculation is needed. Allowed phonon modes have either real or pure imaginary $q_{l}$ values. Modes with real $q_{l}$ have longitudinal waves that are sinusoidal in $z$ direction, and those with pure imaginary $q_{l}$ have longitudinal waves that exponentially decay from the plate surfaces. Figure 1 shows the phonon-dispersion relations with $\sigma=1$ calculated for a free-standing silicon plate. Values of $\rho=2330 \mathrm{~kg} / \mathrm{m}^{3}, \lambda=93.4 \mathrm{GPa}$, and $\mu=51.5 \mathrm{GPa}$ were used. ${ }^{13}$ The horizontal and vertical axes are normalized so that the dispersion relation is valid for any plate thickness, $L_{z}$. The allowed phonon energy for a given in-plane phonon wave vector $Q=|\boldsymbol{Q}|$ is discrete due to phonon confinement, and therefore constitute the branches.

At room temperature, acoustic phonon energy is small compared to the thermal energy, so ADP scattering is approximated as elastic scattering. The transition probability is then given by Fermi's golden rule as

$$
T\left(n \boldsymbol{K}, n^{\prime} \boldsymbol{K}^{\prime}\right)=\frac{\pi D_{\mathrm{ac}}^{2} k_{\mathrm{B}} T_{\mathrm{L}}}{\hbar L_{x} L_{y} v_{l}^{2} \rho} I_{n, n^{\prime}} \delta\left[E_{n^{\prime}}\left(\boldsymbol{K}^{\prime}\right)-E_{n}(\boldsymbol{K})\right],
$$

where $\left(n, n^{\prime}\right)$ are electron quantum numbers of confinement before/after the scattering, $\left(\boldsymbol{K}, \boldsymbol{K}^{\prime}\right)$ are in-plane electron wave vectors, $D_{\text {ac }}$ is the ADP constant, $L_{x} L_{y}$ plate area, $k_{\mathrm{B}}$ the Boltzmann constant, $T_{\mathrm{L}}$ the lattice temperature, and $v_{l}$ the longitudinal sound velocity. Although the deformationpotential interaction has anisotropy in some semiconductors such as $\mathrm{Si}$, it can be treated as isotropic problem by introducing an effective deformation-potential constant. ${ }^{14}$ Such approximation has also been used for studies of electronmodulated acoustic phonons in $\mathrm{Si}$ in a previous report. ${ }^{9}$ The quantity $I_{n, n^{\prime}}$ in Eq. (4) is the form factor defined as

$$
\begin{aligned}
& I_{n, n^{\prime}}(\boldsymbol{Q}) \\
& \quad \equiv \sum_{\sigma, q_{l}} \frac{L_{x} L_{y} v_{l}^{2} \rho}{\omega_{ \pm \boldsymbol{q}}^{2}}\left|\left\langle n^{\prime}\left|\left\{i q_{x} v_{x}(z)+i q_{y} v_{y}(z)+\frac{\partial v_{z}(z)}{\partial z}\right\}\right| n\right\rangle\right|^{2},
\end{aligned}
$$

where $v_{x}(z), v_{y}(z)$, and $v_{z}(z)$ denote $x, y$, and $z$ components of $\boldsymbol{v}(z)$, respectively, $\boldsymbol{Q}$ is given by $\boldsymbol{Q}=\boldsymbol{K}^{\prime}-\boldsymbol{K}$, the bra and ket vectors represent electron wave functions in the confinement $(z-)$ direction, and the summation over $q_{l}$ is taken for all allowed discrete values. Note that Eq. (5) is invariant to the choice of the $x$ and $y$ axes. As long as form-factor calculation is concerned, the $x$ axis can then be reset as parallel to $Q$ without any loss of generality. Using Eq. (3) with $q_{x}=Q$, Eq. (5) reads

$$
\begin{aligned}
I_{n, n^{\prime}}(Q) & =\sum_{\sigma, q_{l}} \rho L_{x} L_{y}\left|C_{l}\right|^{2}\left|\left\langle n^{\prime}\left|\left(e^{i q_{l^{z}}}+\sigma e^{-i q_{l^{z}}}\right)\right| n\right\rangle\right|^{2} \\
& \equiv \sum_{\sigma, q_{l}} K_{n, n^{\prime}}^{\sigma}\left(Q, q_{l}\right) .
\end{aligned}
$$

Thus, the coefficient $\left|C_{l}\right|^{2}$ of the mixed P-SV modes plays an important role in electron-phonon interaction, as expected.

For bulk phonons, the form factor reads

$I_{n, n^{\prime}, \text { bulk }}=\frac{1}{2 \pi} \int_{-\infty}^{\infty}\left|\left\langle n^{\prime}\left|e^{i q_{l^{z}}}\right| n\right\rangle\right|^{2} d q_{l}=\int_{-L_{z} / 2}^{L_{z} / 2}\left|\phi_{n^{\prime}}(z)\right|^{2}\left|\phi_{n}(z)\right|^{2} d z$,

where $\phi_{n\left(n^{\prime}\right)}(z)$ denote electron wave functions before (after) scattering.

\section{B. Sum rules in the form factor}

It is instructive to analyze phonon modes and the form factor at $Q=0$. In such case, the lowest branch in Fig. 1 represents the phonon modes with pure imaginary $q_{l}$, while the other branches give real $q_{l}$. Real $q_{l}$ phonons have $\left|C_{l}\right|^{2}=\left(2 \rho L_{x} L_{y} L_{z}\right)^{-1} \quad$ when $\quad q_{l}=[2 p-(\sigma+1) / 2] \pi / L_{z} \quad$ with $p=1,2,3, \ldots$, and otherwise $\left|C_{l}\right|^{2}=0$. Therefore, the form factor calculated only for real $q_{l}$ reads

$$
\begin{aligned}
& \sum_{\sigma= \pm 1, q_{l} \in \text { real }} K_{n, n^{\prime}}^{\sigma}\left(Q \rightarrow 0, q_{l}\right) \\
& =\sum_{\sigma= \pm 1} \sum_{p=1}^{\infty} \frac{1}{2 L_{z}} \mid\left\langle n^{\prime}\right|\left(e^{i z \pi(2 p-(\sigma+1) / 2) / L_{z}}+\sigma e^{-i z \pi(2 p-(\sigma+1) / 2) / L_{z}}\right) \\
& \times\left.|n\rangle\right|^{2}=\frac{2}{L_{z}} \sum_{s=0}^{\infty}\left\{\left|\left\langle n^{\prime}\left|\cos \left[z \pi(2 s+1) / L_{z}\right]\right| n\right\rangle\right|^{2}\right. \\
& \left.+\left|\left\langle n^{\prime}\left|\cos \left[z \pi(2 s+2) / L_{z}-\pi / 2\right]\right| n\right\rangle\right|^{2}\right\},
\end{aligned}
$$

where $s=p-1$. From the Appendix, assuming that the wave 
function has a value only within $-L_{z} / 2<z<L_{z} / 2$, the following equation holds

$$
\begin{aligned}
\sum_{s=0}^{\infty}\left|\left\langle n^{\prime}\left|\cos \left[z \pi(2 s+a) / L_{z}+b\right]\right| n\right\rangle\right|^{2} \\
=\frac{1}{4} L_{z}\left[e^{2 b i} \int_{-\infty}^{\infty} d z \phi_{n^{\prime}}^{*}(z) \phi_{n}(z) \phi_{n}^{*}(-z) \phi_{n^{\prime}}(-z)\right. \\
\left.\quad+\int_{-\infty}^{\infty} d z\left|\phi_{n^{\prime}}(z)\right|^{2}\left|\phi_{n}(z)\right|^{2}\right],
\end{aligned}
$$

where $a=1$ and $b=0$ for the summation of the first term in Eq. (8), and $a=2$ and $b=-\pi / 2$ for the second term. Consequently,

$$
\begin{aligned}
\sum_{\sigma= \pm 1, q_{l} \in \text { real }} K_{n, n^{\prime}}^{\sigma}\left(Q \rightarrow 0, q_{l}\right) & =\int_{-\infty}^{\infty}\left|\phi_{n^{\prime}}(z)\right|^{2}\left|\phi_{n}(z)\right|^{2} d z \\
& =I_{n, n^{\prime}, \text { bulk }}
\end{aligned}
$$

On the other hand, there is only one pure imaginary $q_{l}$ allowed at $Q=0$, which tends to zero as $Q$ does. For such modes, $\left|C_{l}\right|^{2}=\left\{4 \rho L_{x} L_{y} L_{z}(1+\lambda / \mu)\right\}^{-1}$ for $\sigma=1$, and $\infty$ for $\sigma$ $=-1$. The infinite value results from the fact that the vectors in the first braces in Eq. (3) tends to zero as $Q$ (and therefore $q_{l}$ ) does. The form factor calculated for imaginary $q_{l}$ and $\sigma$ $=1$ is written as

$$
\sum_{q_{l} \in \text { imag }} K_{n, n^{\prime}}^{\sigma=1}\left(Q \rightarrow 0, q_{l}\right)=\frac{1}{4 L_{z}(1+\lambda / \mu)}\left|\left\langle n^{\prime}\left|\left(e^{i q_{l} z}+e^{-i q_{l} z}\right)\right| n\right\rangle\right|^{2}
$$

As $q_{l} \rightarrow 0$ when $Q \rightarrow 0$,

$$
\sum_{q_{l} \in \text { imag }} K_{n, n^{\prime}}^{\sigma=1}\left(Q \rightarrow 0, q_{l}\right)=\frac{1}{L_{z}(1+\lambda / \mu)} \delta_{n, n^{\prime}},
$$

where $\left|\left\langle n^{\prime} \mid n\right\rangle\right|^{2}=\delta_{n, n^{\prime}}$ is used. Similarly, the form factor calculated for imaginary $q_{l}$ and $\sigma=-1$ is written as

$$
\begin{aligned}
\sum_{q_{l} \in \text { imag }} K_{n, n^{\prime}}^{\sigma=-1}(Q \rightarrow & \left.0, q_{l}\right)=\lim _{Q \rightarrow 0}\left[\rho L_{x} L_{y}\left|C_{l}\left(Q, q_{l}(Q)\right)\right|^{2} \mid\left\langle n^{\prime}\right|\right. \\
& \left.\times\left.\left(e^{i q_{l}(Q) z}-e^{-q_{l}(Q) z}\right)|n\rangle\right|^{2}\right] .
\end{aligned}
$$

Unlike the case of $\sigma=1$, care must be taken for the limit calculation. To further calculate, the following asymptotic expression for the lowest phonon branch with $\sigma=-1$ is used:

$$
q_{l}=i\left(1-\frac{c-1}{6 c^{2}} L_{z}^{2} Q^{2}\right) Q,
$$

where $c \equiv 2+\lambda / \mu$. Using the asymptotic form, $\left|C_{l}\left(Q, q_{l}\right)\right|^{2}$ can be written for $Q \ll 1 / L_{z}$ as

$$
\begin{aligned}
& \left|C_{l}\left(Q, q_{l}\right)\right|^{2} \\
& \quad \simeq \frac{\left[(1-2 c) \theta^{4}-12 c^{3}\left(\theta^{2}-6\right)+c^{2} \theta^{2}\left(\theta^{2}+12\right)\right]^{2}}{4 \rho L_{x} L_{y} L_{z}(c-1) \theta^{2}\left(12 c^{2}+(1-c) \theta^{2}\right)\left(6 c^{2}+(1-c) \theta^{2}\right)^{2}},
\end{aligned}
$$

where $\theta \equiv Q L_{z}$. The squared matrix element in Eq. (13) is approximated for $Q \ll 1 / L_{z}$ as

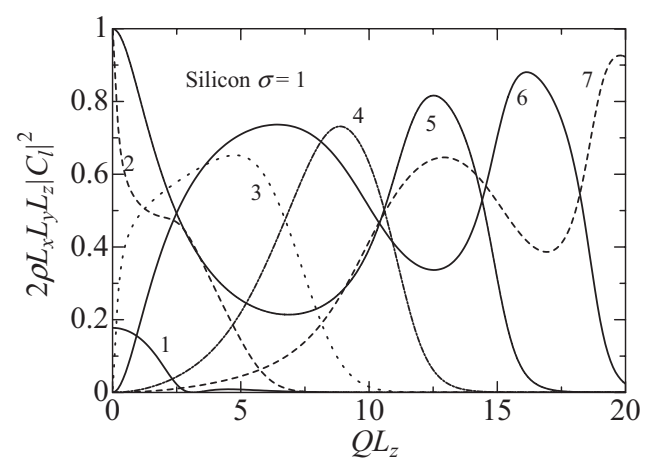

FIG. 2. Squared longitudinal phonon amplitude as a function of $Q L_{z}$ plotted for each dispersion branch on Fig. 1 .

$$
\left|\left\langle n^{\prime}\left|\left(e^{i q_{l}(Q) z}-e^{-q_{l}(Q) z}\right)\right| n\right\rangle\right|^{2} \simeq 4 \theta^{2}\left|\left\langle n^{\prime}|z| n\right\rangle / L_{z}\right|^{2} .
$$

Combining Eqs. (15) and (16), we obtain

$$
\sum_{q_{l} \in \text { imag }} K_{n, n^{\prime}}^{\sigma=-1}\left(Q \rightarrow 0, q_{l}\right)=\frac{1}{L_{z}(1+\lambda / \mu)} \frac{12}{L_{z}^{2}}\left|\left\langle n^{\prime}|z| n\right\rangle\right|^{2} .
$$

Therefore, from Eqs. (12) and (17),

$$
\begin{aligned}
\sum_{\sigma} \sum_{q_{l}} \in \operatorname{imag} & K_{n, n^{\prime}}^{\sigma}\left(Q=0, q_{l}\right) \\
\quad & =\frac{1}{(1+\lambda / \mu) L_{z}}\left(\delta_{n, n^{\prime}}+\frac{12}{L_{z}^{2}}\left|\left\langle n^{\prime}|z| n\right\rangle\right|^{2}\right) .
\end{aligned}
$$

The total form factor at $Q=0$ is finally given by the sum of Eqs. (10) and (18), leading to

$$
I_{n, n^{\prime}}(0)=I_{n, n^{\prime}, \text { bulk }}+\frac{1}{(1+\lambda / \mu) L_{z}}\left(\delta_{n, n^{\prime}}+\frac{12}{L_{z}^{2}}\left|\left\langle n^{\prime}|z| n\right\rangle\right|^{2}\right) .
$$

Thus, the form factor at $Q=0$ is larger than that obtained using bulk phonons due to the modes with longitudinal surface waves. Note that Eqs. (10)-(19) hold for any electron wave functions.

\section{NUMERICAL RESULTS AND DISCUSSIONS}

Figure 2 shows $\left|C_{l}\right|^{2}$ values along each branch shown in Fig. 1, as a function of $Q L_{z}$. The vertical axis is normalized by the amplitude of bulk phonons, $\left(2 \rho L_{x} L_{y} L_{z}\right)^{-1}$. The longitudinal phonon amplitude varies with $Q L_{z}$ because some of the longitudinal phonon amplitude is converted to/from that of transverse phonons, which is referred to as mode conversion. ${ }^{15}$ The amount of mode conversion varies with incident angle of the phonon waves at the interfaces.

Figure 3 shows an example of the form factor numerically calculated for a special case that electron wave functions are those in an infinite square-well potential. Such simplification is valid for the silicon layer thickness less than about $5 \mathrm{~nm}$ and the vertical electric field less than $0.1 \mathrm{MV} / \mathrm{cm} .{ }^{16,17}$ More realistic wave functions can be used, but it is sufficient to use the simplest ones to demonstrate the validity of the equations 


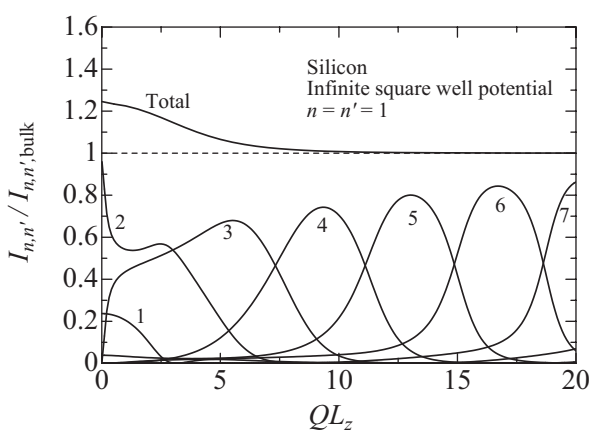

FIG. 3. Form factor of intra-lowest-subband scattering calculated for modulated phonons (denoted as "Total"). The vertical axis is the form factor calculated using modulated phonons, divided by that obtained using bulk phonons. Solid lines with indices show contributions from branches numbered in Fig. 1. The figure is valid for any plate thickness due to normalization of the in-plane phonon wave vector $Q$.

shown above. The solid line denoted as "Total" shows the form factor plotted for intra-lowest-subband scattering ( $n$ $=n^{\prime}=1$ ) as a function of $Q L_{z}$. The form factor is normalized by that of bulk phonons, and the horizontal axis is $Q L_{z}$ so that the figure is valid for any plate thickness. The lines with indices show contributions from each phonon branch shown in Fig. 1. The curves have similar shapes as $\left|C_{l}\right|^{2}$ shown in Fig. 2, proving that the longitudinal phonon amplitude plays a major role in electron-phonon interaction. The total form factor approaches that calculated for bulk phonons at large $Q L_{z}$. At small $Q L_{z}$, on the other hand, the normalized formfactor value is larger than unity. From Eq. (19), the form factor increase at $Q=0$ divided by bulk phonon form factor is written as $\Delta I_{1,1}(0) / I_{1,1, \text { bulk }}=2 /\{3(1+\lambda / \mu)\}$. Note that the angle brackets in Eq. (19) vanishes due to the symmetry of the electron wave functions. For silicon, $\Delta I_{1,1}(0) / I_{1,1, \text { bulk }}$ gives 0.236 . Figure 4 (a) shows the total form factor plotted for different channel materials. The form factor at $Q=0$ will have different values because of the difference in $\lambda / \mu$. Figure 4(b) shows $\Delta I_{1,1} / I_{1,1, \text { bulk }}$ normalized by $\Delta I_{1,1}(0) / I_{1,1, \text { bulk }}$, exhibiting a universal curve regardless of the material as well

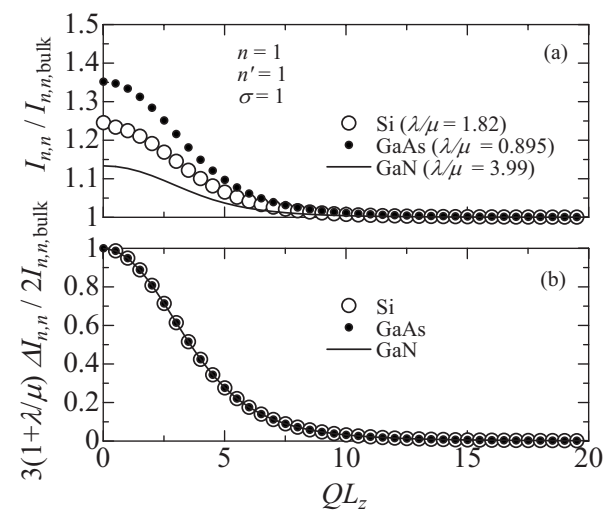

FIG. 4. (a) Normalized form factor of intra-lowest-subband scattering, plotted for different channel materials. (b) Form factor increase from that obtained using bulk phonons, normalized by the values at $Q=0$, showing a universal curve independent of plate material and thickness. as thickness. This universality indicates that the sum rules similar to Eqs. (10)-(19) also exist for nonzero $Q L_{z}$ values, although the formulation might be much more complicated. We confirmed the same universality for $n \geq 2$, as well as for intersubband scattering. The universal form factors are shown by open circles in Figs. 5(a) and 5(b). For intersubband scattering with odd $\left|n-n^{\prime}\right|$, the form factor increase at $Q=0$ comes from the second term in the round bracket in Eq. (19). Considering that the bulk phonon form factor for intersubband scattering is given by $I_{n, n^{\prime} \text {, bulk }}=L_{z}^{-1}$, we obtain 3(1 $+\lambda / \mu) \Delta I_{n, n^{\prime}}(0) / 2 I_{n, n^{\prime}, \text { bulk }}=18\left|\left\langle n^{\prime}|z| n\right\rangle\right|^{2} / L_{z}^{2}$, which gives 0.584 and $3.74 \times 10^{-3}$ for $\left(n, n^{\prime}\right)=(1,2)$ and $(1,4)$, respectively.

Such universality is beneficial for obtaining analytical expressions for electron-phonon scattering rates and mobility. For intrasubband scattering, the universal curve has been shown to be fitted well by ${ }^{10}$

$$
f_{n, n}(\xi) \equiv \frac{3}{2}\left(1+\frac{\lambda}{\mu}\right) \frac{\Delta I_{n, n}(Q)}{I_{n, n, \text { bulk }}}=\frac{4 n^{4} \pi^{2} \tanh (\xi)}{\xi\left(\xi^{4}+5 n^{2} \pi^{2} \xi^{2}+4 n^{4} \pi^{2}\right)},
$$

where $\xi=0.22 Q L_{z}$, which gives good agreement for $1 \leq n$ $\leq 5$. When the assumption of electric quantum limit is valid, analytical formulae of scattering rate and electron mobility calculated using Eq. (20) and electron wave functions in an infinite square-well potential are given by

$$
\begin{aligned}
\frac{1}{\tau_{n, \text { intra }}\left(E_{x y}\right)}= & n_{v} \frac{D_{\mathrm{ac}}^{2} m_{d}^{*} k_{\mathrm{B}} T_{L}}{v_{l}^{2} \rho \hbar^{3}} \frac{1}{L_{z}} \\
& \times\left\{\frac{3}{2}+\frac{1}{1+\lambda / \mu} \frac{4 n^{4} \pi^{2} \tanh (\xi)}{\xi\left(\xi^{4}+5 n^{2} \pi^{2} \xi^{2}+4 n^{4} \pi^{2}\right)}\right\},
\end{aligned}
$$

$$
\begin{aligned}
\mu_{n, \text { intra }}= & \frac{e v_{l}^{2} \rho \hbar^{3}}{n_{v} D_{\mathrm{ac}}^{2} m_{c}^{*} m_{d}^{*} k_{\mathrm{B}} T_{L}} \\
& \times\left[\frac{1}{L_{z}}\left\{\frac{3}{2}+\frac{1}{1+\lambda / \mu} \frac{4 n^{4} \pi^{2} \tanh \left(\xi_{0}\right)}{\xi_{0}\left(\xi_{0}^{4}+5 n^{2} \pi^{2} \xi_{0}^{2}+4 n^{4} \pi^{2}\right)}\right\}\right]^{-1},
\end{aligned}
$$

where $n=1, \quad \xi=0.22 \times 2 \sqrt{m_{c}^{*} E_{x y} / \hbar^{2}} L_{z}, \quad$ and $\quad \xi_{0}=0.22$ $\times 2 \sqrt{m_{c}^{*} k_{\mathrm{B}} T_{e} / \hbar^{2}} L_{z}$. The mobility formula demonstrated reasonable agreement with numerical results for a plate thickness at which the assumption of electric quantum limit holds. ${ }^{10}$ A more compact expression than Eq. (20) is

$$
f_{n, n}(\xi)=\frac{2 n-1}{2 \xi^{5 / 2}+2 n-1},
$$

which gives good agreement for $1 \leq n \leq 3$, but less accuracy than Eq. (20) for $n \geq 4$. Similarly, for intersubband scatterings, the following fitting expressions can be used:

$$
f_{1,2}(\xi)=c_{1} \frac{3}{2 \xi^{5 / 2}+3},
$$




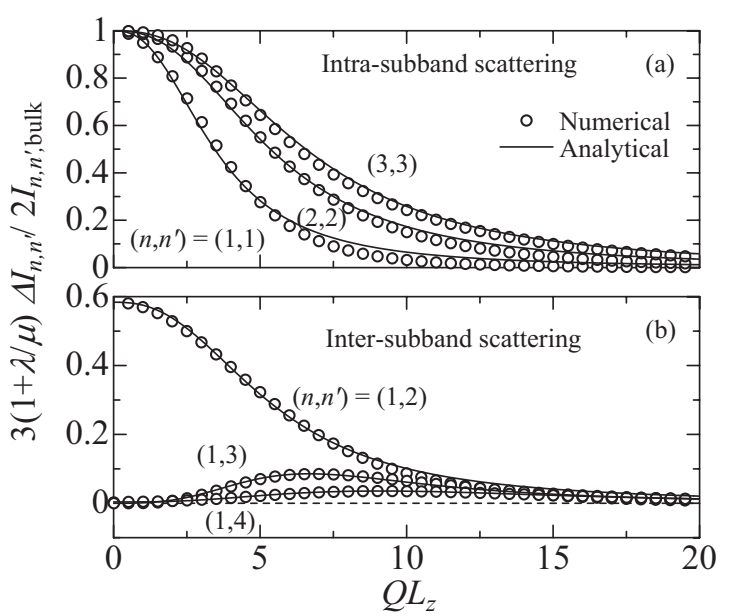

FIG. 5. Universal form factor increase for (a) intra- and (b) intersubband scatterings. Open circles represent numerically obtained universal curves for $\mathrm{Si}, \mathrm{GaAs}$, and GaN. Solid lines show the analytical compact formulae [Eqs. (23)-(26)].

$$
\begin{gathered}
f_{1,3}(\xi)=c_{2}\left(\frac{\xi^{3}}{\xi^{3}+2}+\frac{5}{\xi^{3}+5}-1\right), \\
f_{1,4}(\xi)=c_{3}+c_{4}\left(\frac{\xi^{3}}{\xi^{3}+2}+\frac{5}{c_{5} \xi^{4}+5}-1\right),
\end{gathered}
$$

where $c_{1}=0.584, c_{2}=0.38, c_{3}=3.74 \times 10^{-3}, c_{4}=0.048$, and $c_{5}=0.05$. Comparison with numerical results is shown in Fig. 5, exhibiting excellent agreement.

The increased interaction between electrons and acoustic phonons is due to increase in number of phonon modes having longitudinal phonon vibration. One mechanism is generation of surface modes. Modulated acoustic phonons are either sinusoidal or surface modes, and at near $Q=0$, the form factor increase is merely due to the surface modes as demonstrated in the sum rule, as well as in Fig. 3. Such surface phonons are not included in bulk acoustic phonons because there is no interface of material assumed. Another mechanism is mode conversion. ${ }^{15}$ In three-dimensional bulk phonons, there are two transverse (shear) modes per one longitudinal (pressure) mode due to the degree of freedom. In a free-standing plate, however, such transverse phonons undergo the mode conversion at the interfaces; some of the transverse phonon amplitude is converted to longitudinal phonon amplitude. As a result, virtually all phonon modes can have some longitudinal phonon amplitude, increasing the number of phonon modes contributing to the electronacoustic phonon interaction. Such mode conversion does not occur when the incident angle is zero, which explains the fact that the form factor increase at $Q=0$ is due only to the surface mode. For $Q L_{z} \rightarrow \infty$, phonon waves are not affected by the surfaces at all, so that the form factor becomes identical to that of bulk phonons. The above discussion is also supported by the following argument. Elastic wave theory states that both the surface phonon generation and mode conversion do not occur when $\mu=0$, no shear stress case. As the form factor increase in Eq. (19) also vanishes when $\mu=0$, the form factor increase is attributed to the surface phonon generation and the mode conversion.

It is interesting to note that the form factor does not increase for optical phonon scattering in polar materials. ${ }^{18}$ For optical phonon scattering, dielectric continuum model gives good approximation, which is equivalent to ignoring the transverse components of phonon vibration. As only the longitudinal phonons constitute the orthonormal set of functions, summed contribution to the form factor is unchanged even with phonon modulation due to material interfaces. On the other hand, for acoustic phonon scattering, a mixture of longitudinal and transverse phonons constitutes a normal mode, while only longitudinal components contribute to the electron-phonon scattering. Summed contribution to the form factor can thus be changed due to phonon modulation in the case of acoustic phonon scattering.

\section{CONCLUSION}

In conclusion, insights into modulated acoustic phonons and their interaction with electrons in an ultrathin freestanding semiconductor plate have been presented. The form factor at large $Q L_{z}$ is identical to that calculated for bulk phonons, whereas it is larger at small $Q L_{z}$. The form factor increase is due to increase in number of phonon modes having longitudinal component, which leads to the reduction in the electron mobility. The sum rules of the form factor at $Q=0$ have been presented. When properly normalized, the form factors lie on a universal curve regardless of plate thickness and material.

\section{ACKNOWLEDGMENTS}

This research was partially supported by the Ministry of Education, Science, Sports and Culture, Grant-in-Aid for Young Scientists (B), Grants No. 18760251 and No. 20760221, as well as the Global COE Program "Electronic Devices Innovation." The authors would also like to thank Tatematsu Foundation for financial support.

\section{APPENDIX}

In this appendix, a proof of the following equation is presented:

$$
\begin{aligned}
\sum_{s=0}^{\infty}\left|\left\langle n^{\prime}\left|\cos \left[z \pi(2 s+a) / L_{z}+b\right]\right| n\right\rangle\right|^{2} \\
=\frac{1}{4} L_{z}\left[e^{2 b i} \int_{-\infty}^{\infty} d z \phi_{n^{\prime}}^{*}(z) \phi_{n}(z) \phi_{n}^{*}(-z) \phi_{n^{\prime}}(-z)\right. \\
\left.\quad+\int_{-\infty}^{\infty} d z\left|\phi_{n^{\prime}}(z)\right|^{2}\left|\phi_{n}(z)\right|^{2}\right]
\end{aligned}
$$

where the wave function has a value only within $-L_{z} / 2<z$ $<L_{z} / 2$, and $(a, b)=(1,0)$ or $(2,-\pi / 2)$. By changing the order of the summation and integration in the left-hand side (LHS) of Eq. (A1), we obtain 


$$
\begin{aligned}
\sum_{s=0}^{\infty} \mid & \left.\left\langle n^{\prime}\left|\cos \left[z \pi(2 s+a) / L_{z}+b\right]\right| n\right\rangle\right|^{2} \\
= & \int_{-L_{z} / 2}^{L_{z^{\prime}} / 2} d z \phi_{n^{\prime}}^{*}(z) \phi_{n}(z) \int_{-L_{z / 2}}^{L_{z^{\prime}} / 2} d z^{\prime} \phi_{n}^{*}\left(z^{\prime}\right) \phi_{n^{\prime}}\left(z^{\prime}\right) \\
& \times \sum_{s=0}^{\infty} \cos \left[z \pi(2 s+a) / L_{z}+b\right] \cos \left[z^{\prime} \pi(2 s+a) / L_{z}+b\right]
\end{aligned}
$$

The summation over $s$ is rewritten as

$$
\begin{aligned}
\sum_{s=0}^{\infty} \cos \left[z \pi(2 s+a) / L_{z}+b\right] \cos \left[z^{\prime} \pi(2 s+a) / L_{z}+b\right] \\
=\frac{1}{2} \sum_{s=0}^{\infty} \cos \left[\left(z+z^{\prime}\right) \pi(2 s+a) / L_{z}+2 b\right] \\
+\frac{1}{2} \sum_{s=0}^{\infty} \cos \left[\left(z-z^{\prime}\right) \pi(2 s+a) / L_{z}\right]
\end{aligned}
$$

The first term of the right-hand side (RHS) of Eq. (A3) is calculated as follows:

$$
\begin{aligned}
& \frac{1}{2} \sum_{s=0}^{\infty} \cos \left[\left(z+z^{\prime}\right) \pi(2 s+a) / L_{z}+2 b\right] \\
& \quad=\frac{1}{4} \sum_{s=0}^{\infty}\left[e^{i\left\{\left(z+z^{\prime}\right) \pi(2 s+a) / L_{z}+2 b\right\}}+e^{-i\left\{\left(z+z^{\prime}\right) \pi(2 s+a) / L_{z}+2 b\right\}}\right] \\
& =e^{2 b i} \frac{1}{4} \sum_{s=0}^{\infty}\left[e^{i\left(z+z^{\prime}\right) \pi(2 s+a) / L_{z}}+e^{-i\left(z+z^{\prime}\right) \pi(2 s+a) / L_{z}}\right]
\end{aligned}
$$

where the relation $e^{-4 b i}=1$ has been used. Now, with the help of Fig. 6, the following equation holds for $a=1$ or 2 and arbitral function $f[x]$ :

$$
\sum_{s=0}^{\infty}\{f[(2 s+a)]+f[-(2 s+a)]\}=\sum_{s=-\infty}^{\infty} f[(2 s+a)]-\delta_{a, 2} f[0] .
$$

Therefore, Eq. (A4) leads to

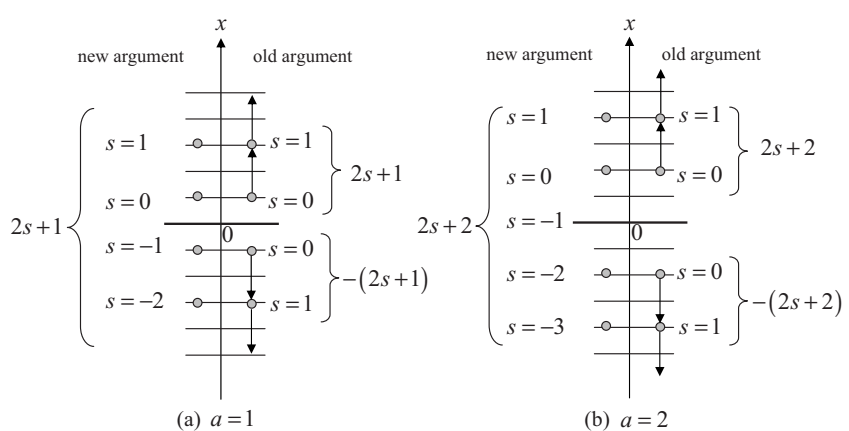

FIG. 6. Schematic illustration of rearranging the summation over the argument $s$ of in Eq. (A5) for (a) $a=1$ and (b) $a=2$.

$$
\begin{aligned}
& \frac{1}{2} \sum_{s=0}^{\infty} \cos \left[\left(z+z^{\prime}\right) \pi(2 s+a) / L_{z}+2 b\right] \\
& \quad=e^{2 b i} \frac{1}{4}\left[\sum_{s=-\infty}^{\infty} e^{i\left(z+z^{\prime}\right) \pi(2 s+a) / L_{z}}-\delta_{a, 2}\right] \\
& =e^{2 b i} \frac{1}{4}\left[e^{i \pi a\left(z+z^{\prime}\right) / L_{z}} \sum_{s=-\infty}^{\infty} e^{i\left(z+z^{\prime}\right) \pi 2 s / L_{z}}-\delta_{a, 2}\right] \\
& \quad=e^{2 b i} \frac{1}{4}\left[\sum_{s=-\infty}^{\infty} e^{i \pi a\left(z+z^{\prime}\right) / L_{z}} \delta\left[\left(z+z^{\prime}\right) / L_{z}-s\right]-\delta_{a, 2}\right]
\end{aligned}
$$

where the following formula is used:

$$
\sum_{s=-\infty}^{\infty} e^{i 2 \pi s x}=\sum_{s=-\infty}^{\infty} \delta(x-s)
$$

Similarly, the second term of the RHS of Eq. (A3) becomes

$$
\begin{aligned}
& \frac{1}{2} \sum_{s=0}^{\infty} \cos \left[\left(z-z^{\prime}\right) \pi(2 s+a) / L_{z}\right] \\
& \quad=\frac{1}{4}\left\{\sum_{s=-\infty}^{\infty} e^{i \pi a\left(z-z^{\prime}\right) / L_{z}} \delta\left[\left(z-z^{\prime}\right) / L_{z}-s\right]-\delta_{a, 2}\right\} .
\end{aligned}
$$

Therefore, Eq. (A3) is rewritten using Eqs. (A6) and (A8) as

$$
\begin{aligned}
& \sum_{s=0}^{\infty} \cos \left[z \pi(2 s+a) / L_{z}+b\right] \cos \left[z^{\prime} \pi(2 s+a) / L_{z}+b\right] \\
& \quad=\frac{1}{4}\left[\sum_{s=-\infty}^{\infty}\left\{e^{2 b i} e^{i \pi a\left(z+z^{\prime}\right) / L_{z}} \delta\left[\left(z+z^{\prime}\right) / L_{z}-s\right]+e^{i \pi a\left(z-z^{\prime}\right) / L_{z}} \delta\left[\left(z-z^{\prime}\right) / L_{z}-s\right]\right\}-\delta_{a, 2}\left(e^{2 b i}+1\right)\right] .
\end{aligned}
$$

The last term in the square bracket vanishes for either case of $(a, b)=(1,0)$ or $(2,-\pi / 2)$. Thus, Eq. (A2) can be written as 


$$
\begin{aligned}
& \sum_{s=0}^{\infty}\left|\left\langle n^{\prime}\left|\cos \left[z \pi(2 s+a) / L_{z}+b\right]\right| n\right\rangle\right|^{2} \\
& \quad=\frac{1}{4} \int_{-\infty}^{\infty} d z \phi_{n^{\prime}}^{*}(z) \phi_{n}(z) \int_{-\infty}^{\infty} d z^{\prime} \phi_{n}^{*}\left(z^{\prime}\right) \phi_{n^{\prime}}\left(z^{\prime}\right) \sum_{s=-\infty}^{\infty}\left\{e^{2 b i} e^{i \pi a\left(z+z^{\prime}\right) / L_{z}} \delta\left[\left(z+z^{\prime}\right) / L_{z}-s\right]+e^{i \pi a\left(z-z^{\prime}\right) / L_{z}} \delta\left[\left(z-z^{\prime}\right) / L_{z}-s\right]\right\} \\
& \quad=\frac{1}{4} L_{z} \sum_{s=-\infty}^{\infty} e^{i \pi a s}\left[e^{2 b i} \int_{-\infty}^{\infty} d z \phi_{n^{\prime}}^{*}(z) \phi_{n}(z) \phi_{n}^{*}\left(s L_{z}-z\right) \phi_{n^{\prime}}\left(s L_{z}-z\right)+\int_{-\infty}^{\infty} d z \phi_{n^{\prime}}^{*}(z) \phi_{n}(z) \phi_{n}^{*}\left(z-s L_{z}\right) \phi_{n^{\prime}}\left(z-s L_{z}\right)\right],
\end{aligned}
$$

where the integrals are formally extended over $(-\infty, \infty)$. As the electron wave functions have a value only in $\left(-L_{z} / 2, L_{z} / 2\right)$, the summation over $s$ in the RHS has a value only at $s=0$, leading to Eq. (A1).

*uno@nuee.nagoya-u.ac.jp

${ }^{1}$ N. Bannov, V. Aristov, V. Mitin, and M. A. Stroscio, Phys. Rev. B 51, 9930 (1995).

${ }^{2}$ N. Bannov, V. Aristov, and V. Mitin, J. Appl. Phys. 78, 5503 (1995).

${ }^{3}$ N. Bannov, V. Mitin, and M. Stroscio, Phys. Status Solidi B 183, 131 (1994).

${ }^{4}$ B. A. Glavin, V. I. Pipa, V. V. Mitin, and M. A. Stroscio, Phys. Rev. B 65, 205315 (2002).

${ }^{5}$ M. Stroscio, J. Appl. Phys. 80, 6864 (1996).

${ }^{6}$ S. M. Komirenko, K. W. Kim, A. A. Demidenko, V. A. Kochelap, and M. A. Stroscio, Phys. Rev. B 62, 7459 (2000).

${ }^{7}$ E. P. Pokatilov, D. L. Nika, and A. A. Balandin, Appl. Phys. Lett. 85, 825 (2004).

${ }^{8}$ E. P. Pokatilov, D. L. Nika, and A. A. Balandin, J. Appl. Phys. 95, 5626 (2004).

${ }^{9}$ L. Donetti, F. Gámiz, J. B. Roldán, and A. Godoy, J. Appl. Phys.
100, 013701 (2006).

${ }^{10}$ S. Uno and N. Mori, Jpn. J. Appl. Phys. 46, L923 (2007).

${ }^{11} \mathrm{~S}$. Uno and N. Mori, Silicon Nanoelectronics Workshop, 4-23, 2007.

${ }^{12}$ A. Soukiassian, W. Tian, D. A. Tenne, X. X. Xi, D. G. Schlom, N. D. Lanzillotti-Kimura, A. Bruchhausen, A. Fainstein, H. P. Sun, X. Q. Pan, A. Cross, and A. Cantarero, Appl. Phys. Lett. 90, 042909 (2007).

${ }^{13}$ M. V. Fischetti, IEEE Trans. Electron Devices 38, 634 (1991).

${ }^{14}$ M. V. Fischetti and S. E. Laux, Phys. Rev. B 48, 2244 (1993).

${ }^{15}$ Karl F. Graff, Wave Motion in Elastic Solids (Dover Publications, Mineola, 1991), Fig. 6.3 on p. 318 and Fig. 8.7 on p. 447.

${ }^{16}$ B. Majkusiak, T. Janik, and J. Walczak, IEEE Trans. Electron Devices 45, 1127 (1998).

${ }^{17}$ M. Shoji and S. Horiguchi, J. Appl. Phys. 85, 2722 (1999).

${ }^{18}$ N. Mori and T. Ando, Phys. Rev. B 40, 6175 (1989). 